\title{
Diastereoselective lower rim (1S)-camphorsulfonylation as the shortest way to the inherently chiral calix[4]arene
}

\author{
Anton V. Yakovenko, ${ }^{1}$ Vyacheslav I. Boyko, ${ }^{1}$ Oksana Danylyuk, ${ }^{2}$ Kinga Suwinska, ${ }^{2}$ Janusz Lipkowski, ${ }^{2}$ Vitaly I. \\ Kalchenko, ${ }^{1, *}$
}

Institute of Organic Chemistry, National Academy of Sciences of Ukraine, Murmanska str. 5, 02660 Kyiv-94, Ukraine and

Institute of Physical Chemistry,

Polish Academy of Sciences, Kasprzaka 44, 01-224 Warsaw, Poland

vik@bpci.kiev.ua

\section{SUPPORTING INFORMATION}

\section{Experimental}

Sulfonylation reaction was carried out in dry solvent, under argon. Melting points were determined on a Boetius apparatus and were uncorrected. ${ }^{1} \mathrm{H}$ NMR $(300 \mathrm{MHz})$ and ${ }^{13} \mathrm{C}$ NMR $(100.1 \mathrm{MHz}$ and $125.8 \mathrm{MHz})$ spectra were recorded on a Varian VXR-300, Varian Mercury - 400 and Bruker-500 instruments. Residual peaks of partially deuterated solvents were used as a standard reference. Specific rotation was measured with Perkin-Elmer Polarimeter 341.

Crystallographic data for the structures in this paper have been deposited into the Cambridge Crystallographic Data Centre as supplementary publication, numbers CCDC 620073 (2a), 620072 (2b). Copies of the data can be obtained, free of charge, on application to CCDC, 12 Union Road, Cambridge CB2 1EZ, UK [fax: 144-(0)1223-336033 or e-mail: deposit@ccdc.cam.ac.uk].

Intensity data were collected at 100(2) K on a Nonius KappaCCD diffractometer using MoK $\alpha$ radiation $(\lambda=$ $0.71073 \AA$ A). Data corrected for Lorentz and polarization effects but not for absorption. The structure was solved and refined using the programs SHELXS-97 (Sheldrick, 1990) and SHELXL-97 (Sheldrick, 1997) respectively. H-atoms were included in geometric positions and refined as 'riding' atoms (except those of hydroxyl groups which were located on Fourier difference maps with positional parameters refined) with isotropic thermal parameters based upon the corresponding bonding carbon atom $\left[\mathrm{U}_{\text {iso }}=1.2 \mathrm{U}_{\mathrm{eq}}\left(\mathrm{U}_{\mathrm{iso}}=1.5 \mathrm{U}_{\mathrm{eq}}\right.\right.$ for $\mathrm{CH} 3$ and $\mathrm{OH}$ hydrogens $\left.)\right]$.

Sulfonylation of monoisopropoxycalix[4]arene 1. To a stirred soulution of calixarene $\mathbf{1}(1.0 \mathrm{~g}, 2.14 \mathrm{mmol})$ in THF/DMF mixture (16/1) a $60 \%$ suspension of sodium hydride in mineral oil was added (0.094 g, $2.36 \mathrm{mmol})$. The reaction mixture was stirred at room temperature for $1 \mathrm{~h}$ and then camphorsulfonyl chloride $(0.58 \mathrm{~g}, 2.31 \mathrm{mmol}) \mathrm{was}$ added as a solution in THF $(2.5 \mathrm{ml})$. Reaction mixture was stirred at room temperature for $18 \mathrm{~h}$. The solvent then was removed under reduced pressure and oily residue was taken up into chloroform and washed with saturated $\mathrm{NH}_{4} \mathrm{Cl}$ solution. Then the organic phase was dried over $\mathrm{Na}_{2} \mathrm{SO}_{4}$. The yield of crude diasteremeric mixture is $83 \%$. Crystallization from benzene-hexane yields $41 \%$ (calculated as yield of reaction) of diastereomer $\mathbf{2 a}$ and $35 \%$ of diastereomer $\mathbf{2 b}$.

25-((1S)-10-camphorsulfonyl)-26-isopropoxy-27,28-dihydroxy-calix[4]arene 2a: M.p. $239-240^{\circ} \mathrm{C} .\left[\mathcal{C O}_{D}^{20}=11.7\right.$ $\left(\mathrm{C}=0.008 \mathrm{M}, \mathrm{CHCl}_{3}\right)$. Calculated for $\mathrm{C}_{41} \mathrm{H}_{44} \mathrm{O}_{7} \mathrm{~S}: \mathrm{C} 72.33, \mathrm{H}$ 6.51, S 4.71, found C 72.51, H 6.21, S 4.49.

${ }^{1} \mathrm{H}$ NMR $\left(\mathrm{CDCl}_{3}, 25^{\circ} \mathrm{C}\right) \delta 0.994$ and 1.214 (camphor Me, 2s, 3H each), 1.495 (camphor CH, m, 1H), 1.594 and $1.731\left(i-\mathrm{Pr} \mathrm{CH}_{3}, 2 \mathrm{~d}, \mathrm{~J}=6.1 \mathrm{~Hz}, 3 \mathrm{H}\right.$ each), 1.822 (camphor $\left.\mathrm{CH}, \mathrm{m}, 1 \mathrm{H}\right), 1.990-2.198$ (camphor CH, m, 3H), 2.481 and 2.655 (camphor $\mathrm{CH}, 2 \mathrm{~m}, 1 \mathrm{H}$ each), 3.386, 3.407, 3.512(e- $\left.\mathrm{ArCH}_{2} \mathrm{Ar}, 3 \mathrm{~d}, \mathrm{~J}=13.5,12.8,13.5 \mathrm{~Hz}, 1 \mathrm{H}, 1 \mathrm{H}, 2 \mathrm{H}\right)$, 3.770 (camphor $\left.\mathrm{OCH}_{2}, \mathrm{~d}, \mathrm{~J}=14.9 \mathrm{~Hz}, 1 \mathrm{H}\right), 4.070\left(\mathrm{a}-\mathrm{ArCH}_{2} \mathrm{Ar}, \mathrm{d}, \mathrm{J}=13.9 \mathrm{~Hz}, 1 \mathrm{H}\right), 4.222(\mathrm{a}-\mathrm{ArCH} \mathrm{Ar} \mathrm{A}, \mathrm{J}=13.5$

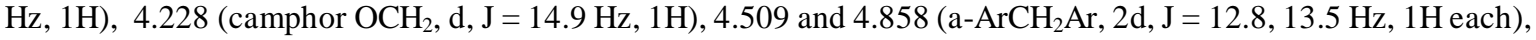
$4.642(i \mathrm{Pr} \mathrm{CH}, \mathrm{sp}, \mathrm{J}=6.1 \mathrm{~Hz}, 1 \mathrm{H}), 6.659(\mathrm{ArH}, \mathrm{m}, 2 \mathrm{H}), 6.824-7.091(\mathrm{ArH}, \mathrm{m}, 10 \mathrm{H}), 8.873$ and $9.717(\mathrm{OH}, 2 \mathrm{~s}, 1 \mathrm{H}$ each).

${ }^{13} \mathrm{C} \mathrm{NMR}\left(\mathrm{CDCl}_{3}, 25^{\circ} \mathrm{C}\right) 19.85,20.11,21.64,21.97,25.11,27.05,31.39,31.87,32.58,33.10,42.59,42.90,48.03$, 48.23, 58.19, 79.61, 120.01, 121.47, 125.77, 127.16, 127.42, 127.77, 127.86, 128.50, 128.55, 128.71, 128.76, $129.25,129.39,129.53,129.78,130.07,134.33,135.21,135.82,136.37,142.68,149.87,149.92,152.4$.

Crystallografic data for compound $2 \mathrm{a} \cdot \mathrm{C}_{6} \mathrm{H}_{6}: \mathrm{C}_{41} \mathrm{H}_{44} \mathrm{O}_{7} \mathrm{~S} \cdot \mathrm{C}_{6} \mathrm{H}_{6}, M r=758.93$, colorless, $0.20 \times 0.10 \times 0.10 \mathrm{~mm}$, orthorhombic, $P 2{ }_{1} 2_{1} 2_{1}, a=11.7745(2), b=16.8129(3), c=19.8021(4) \AA, V=3920.1(1) \AA^{3}, Z=4, \rho_{\text {calc }}=1.286$

${ }^{1}$ Institute of Organic Chemistry, NASU

${ }^{2}$ Institute of Physical Chemistry, PAS 
$\mathrm{g} / \mathrm{cm}^{3}, F_{000}=1616, \theta_{\max }=27.5^{\circ}, \mu=0.136 \mathrm{~mm}^{-1}, 6672$ independent reflections, $5520[\mathrm{I}>2 \sigma(\mathrm{I})] . R=0.035, w R=$ $0.068,(R=0.049, w R=0.070$ for all data $), G o o F=0.961$. Absolute structure parameter $=-0.08(5)($ Flack, H. D. Acta Cryst. 1983, A39, 876-881).

25 - isopropoxy-26-((1S)-10-camphorsulfonyl)-27,28-dihydroxycalix[4]arene 2b M.p. $170-171^{\circ} \mathrm{C} .[\alpha]_{D}^{20}=$ $3.0\left(\mathrm{C}=0.009 \mathrm{M}, \mathrm{CHCl}_{3}\right)$. Calculated for $\mathrm{C}_{41} \mathrm{H}_{44} \mathrm{O}_{7} \mathrm{~S}: \mathrm{C} 72.33, \mathrm{H} 6.51, \mathrm{~S} 4.71$, found C 72.0, H 6.37, S 4.31.

${ }^{1} \mathrm{H} \mathrm{NMR}\left(\mathrm{CDCl}_{3}, 25^{\circ} \mathrm{C}, 300 \mathrm{MHz}\right) \delta 1.036$ and 1.27 (camphor Me, 2s, 3H each), 1.452 (camphor, $\left.\mathrm{m}, 1 \mathrm{H}\right), 1.532$ and 1.748 ( $i$-Pr $\mathrm{CH}_{3}, 2 \mathrm{~d}, \mathrm{~J}=6.1 \mathrm{~Hz}, 3 \mathrm{H}$ each), 1.958 (camphor $\mathrm{CH}, \mathrm{m}, 1 \mathrm{H}$ ), 2.06-2.182 (camphor $\mathrm{CH}, \mathrm{m}, 3 \mathrm{H}$ ), 2.433 and 2.606 (camphor $\mathrm{CH}, 2 \mathrm{~m}, 1 \mathrm{H}$ each), 3.448, 3.491, 3.554, $3.575\left(\mathrm{e}-\mathrm{ArCH}_{2} \mathrm{Ar}, 4 \mathrm{~d} \mathrm{~J}=13.7,13.1,13.9,13.1\right.$ $\mathrm{Hz}, 4 \mathrm{H}), 3.586$ (camphor $\left.\mathrm{OCH}_{2}, \mathrm{~d}, \mathrm{~J}=14.8 \mathrm{~Hz}, 1 \mathrm{H}\right), 4.081\left(\mathrm{a}-\mathrm{ArCH}_{2} \mathrm{Ar}, \mathrm{d}, \mathrm{J}=13.9 \mathrm{~Hz}, 1 \mathrm{H}\right), 4.094($ camphor OCH , d, J = 14.8 Hz, 1H), 4.227, 4.612, $4.791\left(\mathrm{a}-\mathrm{ArCH}_{2} \mathrm{Ar}, 3 \mathrm{~d}, \mathrm{~J}=13.7,13.1,13.1 \mathrm{~Hz}, 3 \mathrm{H}\right), 4.668(i \operatorname{Pr} \mathrm{CH}, \mathrm{sp}, \mathrm{J}=6.1$ $\mathrm{Hz}, 1 \mathrm{H}), 6.647$ (ArH, m, 2H), 6.833 (ArH, m, 1H) 6.924-7.117 (ArH, m, 8H), 7.245 (ArH, m, 1H), 8.938 and 9.799 $(\mathrm{OH}, 2 \mathrm{~s}, 1 \mathrm{H}$ each).

Crystallografic data for compound $2 \mathbf{b} \cdot \mathrm{CHCl}_{3}: \mathrm{C}_{41} \mathrm{H}_{44} \mathrm{O}_{7} \mathrm{~S}^{\cdot} \mathrm{CHCl}_{3}: \mathrm{Mr}=800.19$, colorless, $0.40 \times 0.15 \times 0.13 \mathrm{~mm}$, monoclinic, $P 2_{1}, a=11.1455(2), b=15.2453(3), c=11.5346(2) \AA, \beta=95.8198(8)^{\circ}, V=1949.82(6) \AA^{3}, Z=2, \rho_{\text {calc }}$ $=1.363 \mathrm{~g} / \mathrm{cm}^{3}, F_{000}=840, \theta_{\max }=26.4^{\circ}, \mu=0.339 \mathrm{~mm}^{-1}, 4073$ independent reflections, $3482[\mathrm{I}>2 \sigma(\mathrm{I})] . R=0.042$, $w R=0.103,(R=0.056, w R=0.108$ for all data $), G o o F=1.064$. Absolute structure parameter $=0.11(7)($ Flack, $\mathrm{H}$. D. Acta Cryst. 1983, A39, 876-881).

5,11-dibromo-25-((1S)-10-camphorsulfonyl)-26-isopropoxy-27,28-dihydroxy-calix[4] arene 3. To a solution of camphosulfonylcalixarene $\mathbf{2 a}(0.155 \mathrm{~g}, 0.228 \mathrm{mmol})$ in dry acetone $(6 \mathrm{ml})$ a freshly crystallized bromosuccineimide $(0.162 \mathrm{~g}, 0.91 \mathrm{mmol})$ was added. Yellow solution has been stirred overnight at room temperature. Solvent was then removed and yellow residue was taken up in hot water, mashed and then filtered off. The crude product was recrystallized from benzene-hexane mixture. Yield $94 \%$. M.p. $215-216^{\circ} \mathrm{C} .[\alpha]_{D}^{20}=9.9(\mathrm{C}=$ $0.006 \mathrm{M}, \mathrm{CHCl}_{3}$ ). Calculated for $\mathrm{C}_{41} \mathrm{H}_{42} \mathrm{Br}_{2} \mathrm{O}_{7} \mathrm{~S}$ : $\mathrm{Br} 19.06$, found $\mathrm{Br} 20.08$.

${ }^{1} \mathrm{H}$ NMR $\delta 0.981$ and 1.203 (camphor Me, 2s, 3H each), 1.478 (camphor $\left.\mathrm{CH}, \mathrm{m}, 1 \mathrm{H}\right), 1.556$ and $1.729\left(i-\mathrm{Pr} \mathrm{CH}_{3}\right.$, 2d, J = 6.1 Hz, 3H each), 1.790 (camphor $\mathrm{CH}, \mathrm{m}, 1 \mathrm{H}$ ), 1.984-2.173 (camphor $\mathrm{CH}, \mathrm{m}, 3 \mathrm{H}$ ), 2.467 and 2.638 (camphor $\mathrm{CH}, 2 \mathrm{~m}, 1 \mathrm{H}$ each), 3.288, 3.374, 3.479, 3.540 (e-ArCH $\mathrm{Ar}_{2} \mathrm{Ar}, 4 \mathrm{~d}, \mathrm{~J}=14.3,12.1,14.3,13.2 \mathrm{~Hz}, 1 \mathrm{H}$ each), 3.645 (camphor $\left.\mathrm{OCH}_{2}, \mathrm{~d}, \mathrm{~J}=14.9 \mathrm{~Hz}, 1 \mathrm{H}\right), 4.032\left(\mathrm{e}-\mathrm{ArCH} \mathrm{H}_{2} \mathrm{Ar}, \mathrm{d}, \mathrm{J}=13.7 \mathrm{~Hz}, 1 \mathrm{H}\right), 4.121-4.220\left(\mathrm{e}-\mathrm{ArCH}_{2} \mathrm{Ar}\right.$ and camphor $\left.\mathrm{OCH}_{2}, 2 \mathrm{~d}, \mathrm{~J}=13.9,14.9 \mathrm{~Hz}, 2 \mathrm{H}\right), 4.465\left(\mathrm{e}-\mathrm{ArCH}_{2} \mathrm{Ar}, \mathrm{d}, \mathrm{J}=12.4 \mathrm{~Hz}, 1 \mathrm{H}\right), 4.642(i \mathrm{Pr} \mathrm{CH}, \mathrm{sp}, \mathrm{J}=6.1 \mathrm{~Hz}$, $1 \mathrm{H}), 4.831\left(\mathrm{e}-\mathrm{ArCH}_{2} \mathrm{Ar}, \mathrm{d}, \mathrm{J}=13.2 \mathrm{~Hz}, 1 \mathrm{H}\right), 6.897-7.279$ (ArH protons, m, 10H), 8.717 and $9.903(\mathrm{OH}, 2 \mathrm{~s}, 1 \mathrm{H}$ each).

5,11-dibromo-26-isopropoxy-27,28,25-trihydroxycalix[4]arene 4. To a suspension of calixarene $3(0.06 \mathrm{~g}$, $0.0715 \mathrm{mmol})$ in methanol $(1.5 \mathrm{ml})$ a solution of $40 \%$ of $\mathrm{KOH}(0.035 \mathrm{ml})$ was added. Reaction mixture was refluxed for $2.5 \mathrm{~h}$. The color of the solution turns to dark yellow. Cooled reaction mixture was diluted with $1 \mathrm{M} \mathrm{HCl}$ $(25 \mathrm{ml})$. Yellowish residue was filtered off, then dissolved in benzene and washed with saturated $\mathrm{NH}_{4} \mathrm{Cl}$ water solution and then water. Benzene layer was dried over $\mathrm{Na}_{2} \mathrm{SO}_{4}$ and then evaporated to yield $73 \%$ of 4 as pale beige crystals. M.p. $>250^{\circ} \mathrm{C}$ (decomp.). $[\alpha]_{D}^{20}=16.0\left(\mathrm{C}=0.007 \mathrm{M}, \mathrm{CHCl}_{3}\right)$. Calculated for $\mathrm{C}_{31} \mathrm{H}_{28} \mathrm{Br}_{2} \mathrm{O}_{4}$ : C 59.60, $\mathrm{H} 4.52$, $\mathrm{Br} 25.59$, found $\mathrm{C} 58.82, \mathrm{H} 4.31, \mathrm{Br} 25.12$.

${ }^{1} \mathrm{H}$ NMR $\left(\mathrm{CDCl}_{3}, 25^{\circ} \mathrm{C}, 300 \mathrm{MHz}\right) \delta 1.552$ and $1.623\left(i-\mathrm{Pr} \mathrm{CH}_{3}, 2 \mathrm{~d}, \mathrm{~J}=6.1 \mathrm{~Hz}, 3 \mathrm{H}\right.$ each), 3.312-3,511 (e$\left.\mathrm{ArCH}_{2} \mathrm{Ar}, 4 \mathrm{~d}, \mathrm{~J}=13.7,13.2,14.3,13.2 \mathrm{~Hz}, 4 \mathrm{H}\right), 4.188,4.312,4.461\left(\mathrm{a}-\mathrm{ArCH}_{2} \mathrm{Ar}, 3 \mathrm{~d}, \mathrm{~J}=13.7,13.2,13.2 \mathrm{~Hz}, 2 \mathrm{H}\right.$, $1 \mathrm{H}, 1 \mathrm{H}), 4.551(\mathrm{Pr} \mathrm{CH}, \mathrm{sp}, \mathrm{J}=6.1 \mathrm{~Hz}, 1 \mathrm{H}), 6.689-7.186$ (ArH protons, m, 10H), 9.180, 9.383, $9.725(\mathrm{OH}, 3 \mathrm{~s}, 1 \mathrm{H}$ each).

${ }^{13} \mathrm{C} \mathrm{NMR}\left(\mathrm{CDCl}_{3}, 25^{\circ} \mathrm{C}\right) 21.65,21.81,31.17,31.35,31.59,31.86,79.45,112.19,113.63,121.39,125.91,126.05$, $126.12,127.28,128.10,128.97,129.19,129.51,129.71,130.17,130.35,130.76,130.86,131.13,131.28,131.55$, $134.36,134.45,148.48,149.25 ; 150.38$. 


\section{${ }^{1} \mathrm{H}$ and ${ }^{13} \mathrm{C}$ NMR spectra of the compound 2a}

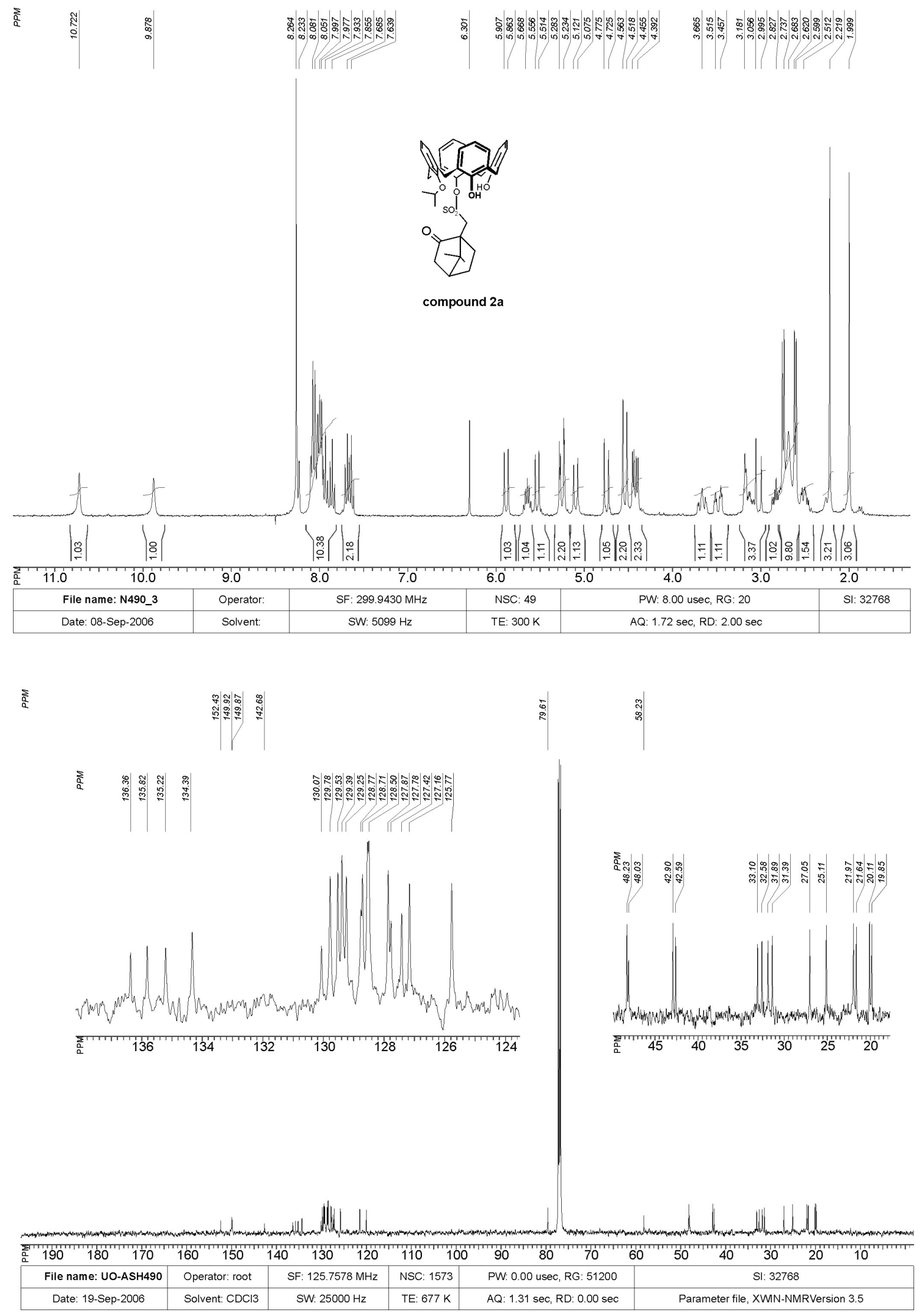




\section{${ }^{1} \mathrm{H}$ NMR spectrum of the compound $\mathbf{2 b}$}
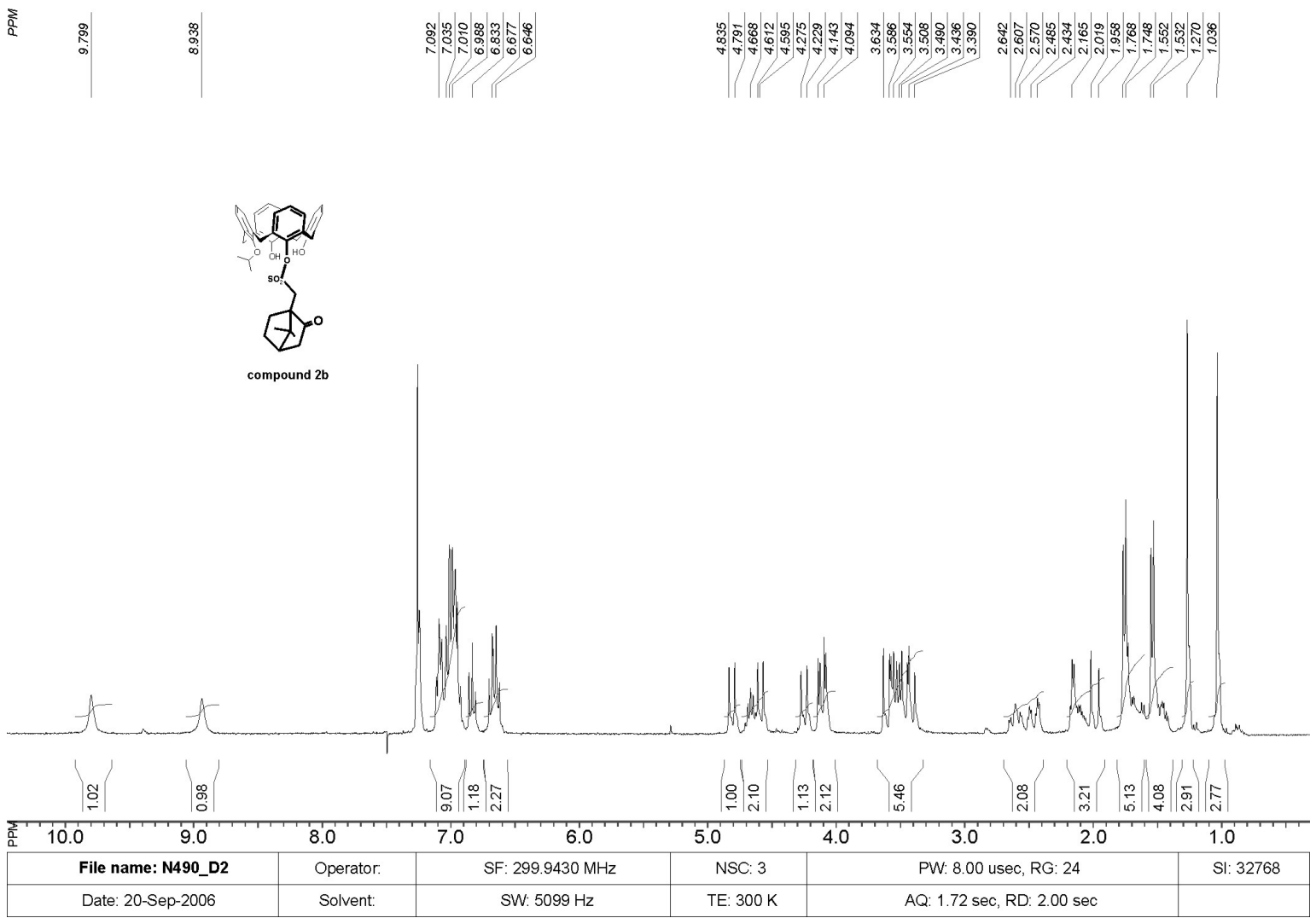


\section{${ }^{1} \mathrm{H}$ NMR spectrum of the compound 3}

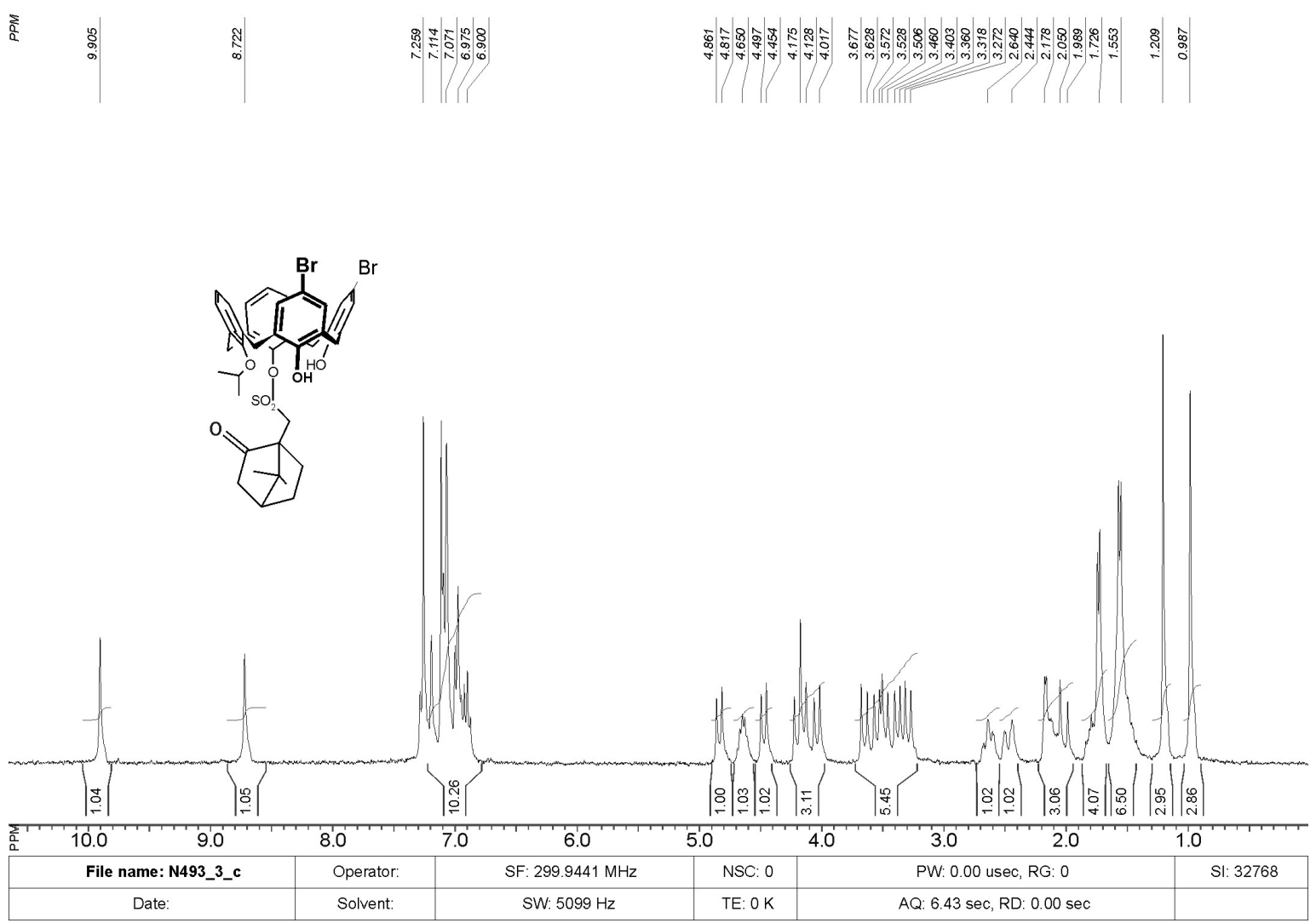




\section{${ }^{1} \mathrm{H}$ and ${ }^{13} \mathrm{C}$ NMR spectra of the compound 4}
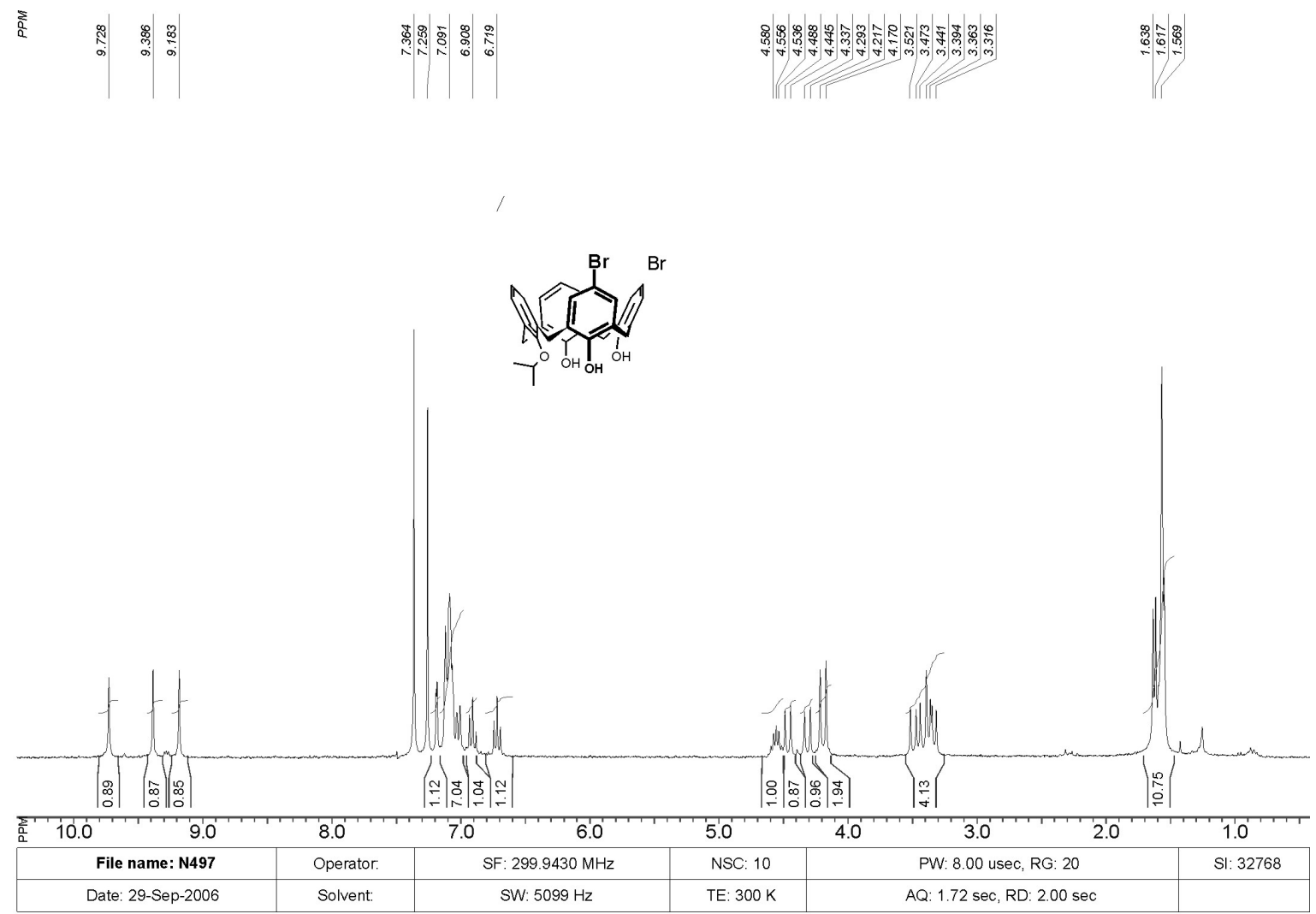

홍
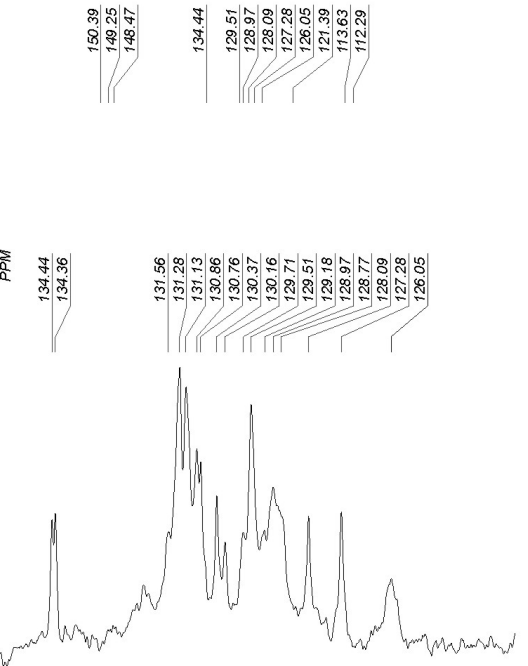

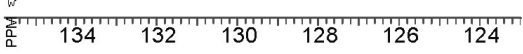

言
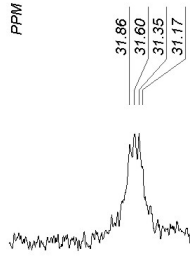

क्ञाता 35

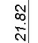 \\ $\stackrel{\infty}{\mathrm{i}}$}

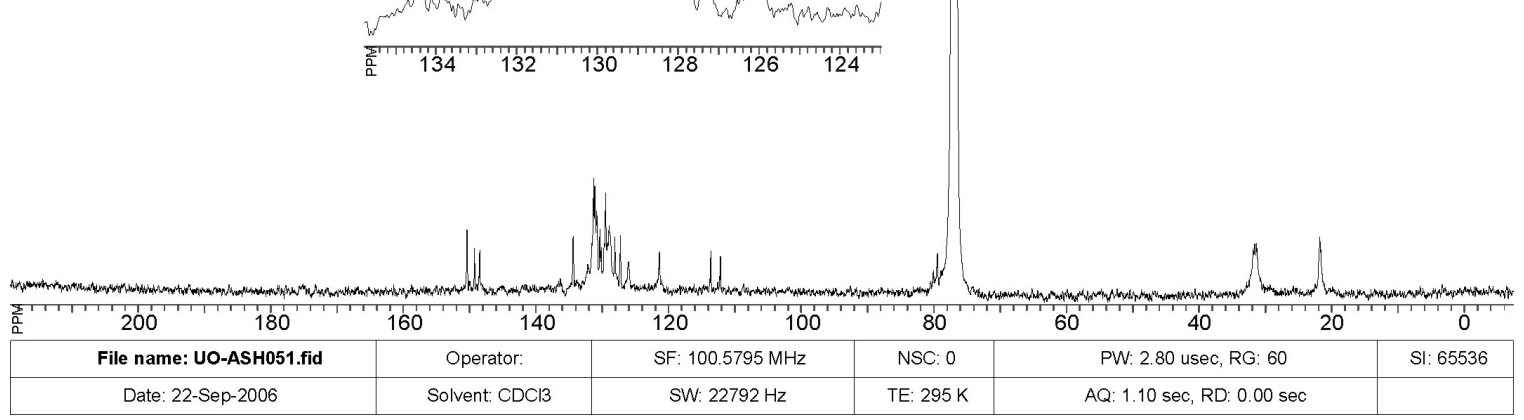

